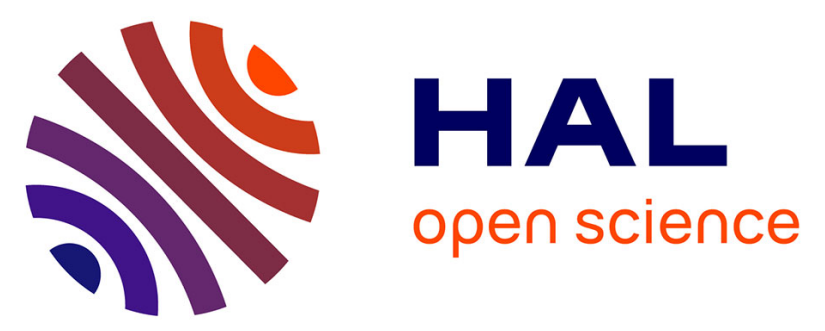

\title{
Self-organization Properties of a GPCR-Binding Peptide with a Fluorinated Tail Studied by Fluorine NMR Spectroscopy
}

Capucine Jourdain de Muison, Sridevi M. Ramanoudjame, Lucie Esteoulle, Claude Ling, Germain Brou, Nicolas Anton, Thierry Vandamme, Marc-André

Delsuc, Dominique Bonnet, Bruno Kieffer

\section{To cite this version:}

Capucine Jourdain de Muison, Sridevi M. Ramanoudjame, Lucie Esteoulle, Claude Ling, Germain Brou, et al.. Self-organization Properties of a GPCR-Binding Peptide with a Fluorinated Tail Studied by Fluorine NMR Spectroscopy. ChemBioChem, 2020, 22 (4), pp.657-661. 10.1002/cbic.202000601 . hal-03090942

\section{HAL Id: hal-03090942 \\ https://hal.science/hal-03090942}

Submitted on 10 Oct 2021

HAL is a multi-disciplinary open access archive for the deposit and dissemination of scientific research documents, whether they are published or not. The documents may come from teaching and research institutions in France or abroad, or from public or private research centers.
L'archive ouverte pluridisciplinaire HAL, est destinée au dépôt et à la diffusion de documents scientifiques de niveau recherche, publiés ou non, émanant des établissements d'enseignement et de recherche français ou étrangers, des laboratoires publics ou privés. 
Self-organization properties of a GPCR binding peptide incorporating fluorinated tail studied by fluorine NMR

Capucine Jourdain de Muizon ${ }^{[a][b]}$, Srid'evi M. Ramanoudjame ${ }^{[c]}$, Lucie Esteoulle $^{[\mathrm{c}]}$, Claude Ling ${ }^{[\mathrm{a}]}$, Germain Brou ${ }^{[\mathrm{d}]}$, Nicolas Anton ${ }^{[\mathrm{d}]}$, Thierry Vandamme $^{[\mathrm{d}]}$, Marc-Andr'e Delsuc ${ }^{[\mathrm{a}][\mathrm{b}]}$, Dominique Bonnet ${ }^{*}[\mathrm{c}]$, Bruno Kieffer*[a]

[a] C. Ling, B. Kieffer

Departement of Integrative Structural Biology, IGBMC

Universit'e de Strasbourg, INSERM U596, CNRS UMR7104

1, rue Laurent Fries, Illkirch, France

E-mail: kieffer@igbmc.fr

[b] C. Jourdain de Muizon, M.A. Delsuc

CASC4DE Le Lodge, 20, Avenue du Neuhof, 67100 Strasbourg, France

[c] S.M. Ramanoudjame, L. Esteoulle, D. Bonnet

Laboratoire d'Innovation Th'erapeutique, LabEx MEDALIS Université de Strasbourg, CNRS UMR 7200

Faculté de Pharmacie, 74 route du Rhin, 67401 Illkirch-Graffenstaden, France E-mail: dbonnet@unistra.fr

[d] G. Brou, N. Anton, T. Vadamme

Conception et Applications de Molécules Bioactives

University of Strasbourg, CNRS UMR 7199

Faculté de Pharmacie, 74 route du Rhin, 67401 Illkirch-Graffenstaden, France

Supporting information for this article is given via a link at the end of the document.

Abstract: The conjugation of the bioactive apelin-17 peptide with a fluorocarbon chain results in higher potency and self-organisational property of the peptide into micelles. Fluorine NMR studies show that fluoropeptide's micelles are mono-disperse while proton NMR indicates that the peptide moiety remains largely disordered despite micellization. A very fast exchange rate is measured between the free and micellar states of the peptide enabling to estimate the number of molecules present in the micelle as two hundred, in agreement with values found by Dynamic Light Scattering measurements.

Chemically modified peptides offer great opportunities for developing novel therapeutic strategies and associated technologies have been considerably extended over the past few years. ${ }^{[1]}$ Among those, the insertion of non-natural amino-acids containing fluorine atoms gains increasing interests due to the distinct chemical properties conferred by the fluorine associated with the ability to get a detailed description of these properties using ${ }^{19} \mathrm{~F}$ NMR. ${ }^{[2]}$ The substitution of natural amino-acids by their fluorinated variants or the addition of trifluoromethyl groups at the protein surface have indeed recently expanded the panel of protein NMR 
technics to provide insights on G Protein Coupled Receptor (GPCR) function, ${ }^{[3]}$ investigate protein-ligand interactions, ${ }^{[4]}$ measure an enzymatic activity ${ }^{[5]}$ or observe protein folding inside living Escherichia coli cells. ${ }^{[6]}$ While these methods see fluorine as a label and assume a minimal effect of the fluorine substitution on the protein state, other applications take advantage of the fluorine properties to perturb the conformation and dynamics of the molecular system in order to gain insights on the related molecular mechanisms. One compelling example is prolines with one of the two geminal $\mathrm{H} \gamma$ hydrogens substituted by a fluorine atom, a modification that induces a bias of the ring conformation and the cistrans equilibrium of the X-Pro peptide bond. ${ }^{[7]}$ This property has been used to investigate the role of hydroxyproline in the self-association mechanism of collagen ${ }^{[8]}$ and the human amyloidogenic $\beta 2$-microglobulin. ${ }^{[9]}$

Another way to modulate biological activities of peptides and proteins is the conjugation with a fluorocarbon chain (F-chain) to one extremity. Indeed, due to their very specific properties and their ability to self-assemble in aqueous solution, the addition of F-chains to organic molecules changes their physical and chemical properties. Thereby, the conjugation of F-chain to insulin has been shown to have an impact on the native hexamer structure and could represent a new way to control self-assembly of proteins and their in vivo stabilities. ${ }^{[10]}$ The addition of F-chain to peptides was applied in the development of vaccines as well. The authors recently demonstrated that the presence of $\mathrm{F}$-chain in peptides derived from HIV, influenza and hepatitis $\mathrm{C}$ virus increased their cellular immunogenicity by controlling their ability to form micelles in solution. ${ }^{[11]}$ They also showed that the conjugation of therapeutic peptides targeting GPCR with F-chain provided a promising approach to enhance their plasma stability and in vivo activity. ${ }^{[12]}$ This approach has been successfully applied to apelin-17. This peptide together with its cognate GPCR receptor dubbed APJ ${ }^{[13]}$ has emerged as novel targets for the treatment of cardiovascular diseases. ${ }^{[14]}$ However, the low stability of apelin-17 in plasma precluded its therapeutic development. To circumvent this limitation, a perfluoroundecanoic acyl group (F8-chain) was incorporated to the N-terminal part of apelin-17 (LIT01-196) resulting in a dramatic enhancement of its half-life in plasma, as well as its efficacy on the cardiac contractility and arterial blood pressure on rat model. ${ }^{[12]}$ For all those applications, it is crucial to characterize and to monitor the self-organization of fluoropeptides in order to strictly control their biological activities.

Thanks to its ability to describe both dynamical and structural features of micelles without requesting any chemical modifications, fluorine NMR is best suited to study the properties of fluorosurfactants. ${ }^{[15]}$ Hence, NMR was successfully used to study micellization of gemini surfactants or perfluorooctylsulfonates. ${ }^{[16]}$ In this paper, using Fluorine NMR as a main investigation tool, we report for the first time on the influence of F8-chain addition to apelin-17 on both the structural and self-assembling properties of the resulting fluoroapelin-17 (F8-Ap). 
A

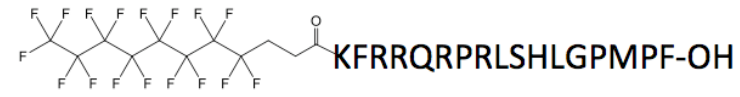

B

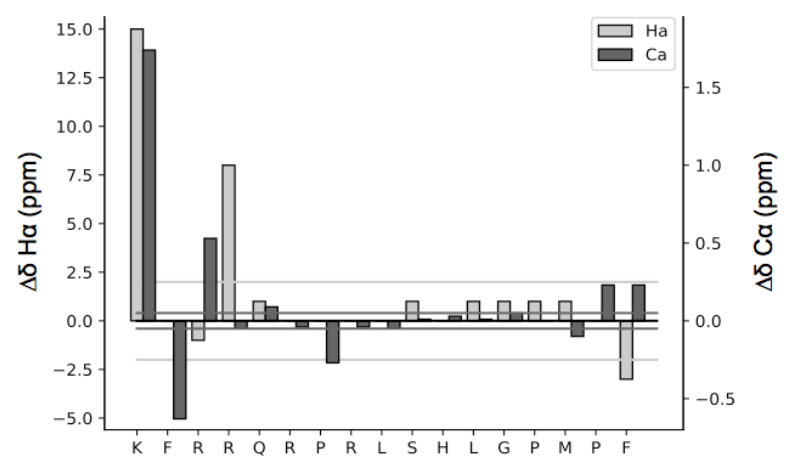

Figure 1. Chemical structure of fluorocarbon conjugated apelin-17 (F8-Ap) (A). Chemical shift differences between the perfluorinated and the natural Apelin-17 peptide for the $\mathrm{H} \alpha$ (grey) and $\mathrm{C} \alpha$ (black) nuclei measured in water and $298 \mathrm{~K}$ (B).

To assess the potential structural changes induced by the addition of the F8-chain at the N-terminal position of apelin-17, preliminary proton NMR spectra of both peptides dissolved in PBS buffer were first recorded at $100 \mu \mathrm{M}$ and $420 \mu \mathrm{M}$ of peptide concentrations. At $420 \mu \mathrm{M}$, the resonances of F8-Ap display significant line broadening while those of apelin-17 remains sharp (data not shown). In order to compare structural propensities of the two peptides, homonuclear ROESY, TOCSY and ${ }^{1} \mathrm{H}^{13} \mathrm{C}$ HSQC spectra of apelin-17 and F8-Ap were recorded at 2 $\mathrm{mM}$ in water and $298 \mathrm{~K}$, where no line broadening is observed. Frequencies of proton and carbon nuclei were obtained using sequential assignment (Figure S1 and Table S1). The chemical shifts of $\mathrm{H}_{\alpha}, \mathrm{H}_{\beta}$ and the corresponding carbon nuclei that offer sensitive probes of the local peptide conformation were used to characterize the potential conformational differences between the two peptides (Figure 1). The condensation of the F8-chain to apelin-17 induces significant chemical shifts changes for the first four N-terminal residues (KFFR, Figure 1A), while the C-terminal display similar chemical shifts.

The differences observed for the first four residues are due to the substitution of the free NH3 group by a peptide bond and probably a reorientation of the aromatic ring of the phenylalanine upon fluorine-pi interaction with the F8chain. ${ }^{[17]}$ The chemical shifts of other amino acids measured for both peptides are very similar and close to theoretical values obtained for a random coil peptide as computed by the Potenci software ${ }^{[18]}$ indicating a lack of secondary structure. Those results are in accordance with previous NMR studies on the structure of apelin-17 by NMR highlighting a predominantly random coil character of the peptide. ${ }^{[19]}$ Thereby, we show here that the incorporation of the F8-chain onto peptide does not impact the structure of apelin- 17 . 

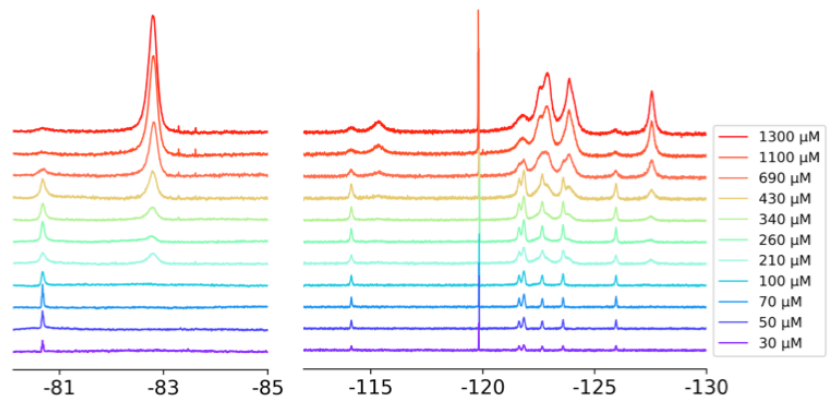

${ }^{19} \mathrm{~F}(\mathrm{ppm})$

Figure 2. $1 \mathrm{D}^{19} \mathrm{~F}$ spectrum of F8-Ap in PBS buffer as a function of total peptide concentration $\mathrm{C}_{0}$ indicated in the right panel. The trifluoromethyl resonance used for quantification is shown in the left panel.

The self-association property of the F8-Ap was then studied by recording fluorine spectra for peptide concentrations ranging from $30 \mu \mathrm{M}$ to $1.3 \mathrm{mM}$ in phosphate buffer. Across this range of concentrations, the fluorine peaks experienced a constant broadening and an additional set of resonances arose for concentrations above $200 \mu \mathrm{M}$, indicating the presence of a second population of molecules in the solution (Figure 2). Owing to their significantly larger line-widths, these resonances were assigned to a pool of peptides organized into micelles. Integrating the trifluoromethyl resonances at -82.8 and $-80.66 \mathrm{ppm}$ was used to measure the relative fractions of the self-organized and the monomer forms of the peptides, respectively. Assuming that the fraction of the monomers $\mathrm{f}_{\text {mono }}$ is described by the following fraction

$$
f_{\text {mono }}=\frac{\mathrm{CMC}}{C_{0}}(1)
$$

where $\mathrm{C}_{0}$ is the total concentration of peptide and $\mathrm{CMC}$, the Critical Micellar Concentration. ${ }^{[16 \mathrm{~b}]}$ The latter is usually determined from the dependence of the monomeric fraction upon the inverse of the total peptide concentration (Figure S2). This analysis led to a CMC value of $80 \mu \mathrm{M}$, in full agreement with the $\mathrm{CMC}$ of $84 \mathrm{\mu M}$ determined by surface tension using a drop tensiometer (Figure S3). This value is significantly lower than the CMC reported for perfluorooctylsulfonates surfactants (in the $\mathrm{mM}$ range), indicating a contribution of the peptide moiety to the interactions within the micelle. Indeed, we observed a large dependence of the fluorine line broadening upon phosphate concentration as expected from the sensitivity of self-association properties of surfactant molecules on ionic strength. ${ }^{[20]}$ In the particular case of F8-Ap, screening electrostatic interactions between the positively charged residues neighbouring the F8 chain may be critical to modulate the peptide self-association properties.

The observation of cross-peaks between the two sets of resonances in a fluorine NOESY spectrum recorded at a concentration of $200 \mu \mathrm{M}$ of F8-Ap indicated that exchange between the monomeric and the micellar states occured in the millisecond time scale (Data not shown). The F8 fluoromethyl group experi- 
enced a chemical shift variation of $2.1 \mathrm{ppm}(1200 \mathrm{~Hz})$ between the two states. This remarkable shift enabled the selective manipulation of the corresponding populations of spins using frequency selective soft pulses (Figure S3). A selective inversion of the fluoromethyl broad peak at $-82.8 \mathrm{ppm}$ resulted into a fast decay of the resonance at $-80.66 \mathrm{ppm}$ corresponding to the monomeric form of the peptide (Figure 3). After $10 \mathrm{~ms}$, the signal became negative before a slow recovery, indicating that the magnetization transfer between the micelle and the monomer resonances through chemical exchange is significantly faster that the longitudinal relaxation rate $\left(\mathrm{R}_{1}\right)$ of the fluoromethyl resonance of the monomeric peptide.

Figure 3. Selective inversion of the trifluoromethyl signal at $-82.8 \mathrm{ppm}$ (blue dots) leads to fast magnetization transfer to the signal at $-80.5 \mathrm{ppm}$ (red dots) corresponding to the tri-fluoromethyl of the free peptide. Fitting of the peak intensities using a three exponentials model allows the determination of the selective $R_{1}$ of the fluoromethyl in both states and the exchange rate between these states

Fitting the time evolution of signal intensities with a three exponentials model accounting for two specific longitudinal relaxation and the chemical exchange rates provided values of $173 \pm 5 \mathrm{~s}^{-1}$ for the exchange rate between the monomeric and the micellar forms, and $7.5 \pm 1.8 \mathrm{~s}^{-1}$ and $1.9 \pm 0.6 \mathrm{~s}^{-1}$ for fluoromethyl's $\mathrm{R}_{1}$ in the monomeric and micellar environment, respectively. As expected, the rate of chemical exchange is significantly slower than frequency difference between the two states $(1200 \mathrm{~Hz})$, explaining why no chemical shift difference is observed upon variation of the total peptide concentration. The lower $R_{1}$ value observed for the fluoromethyl resonance in the micellar state is consistent with a longer correlation time expected in micelles and the expected evolution of the dipolar relaxation upon increasing correlation time. The micelle dissociation rate obtained from the fit corresponded to a mean lifetime of $15.6 \mathrm{~ms}$, a value significantly longer than the 4.2 us observed for perfluorooctylsulfonates. ${ }^{[16 \mathrm{~b}]}$ 


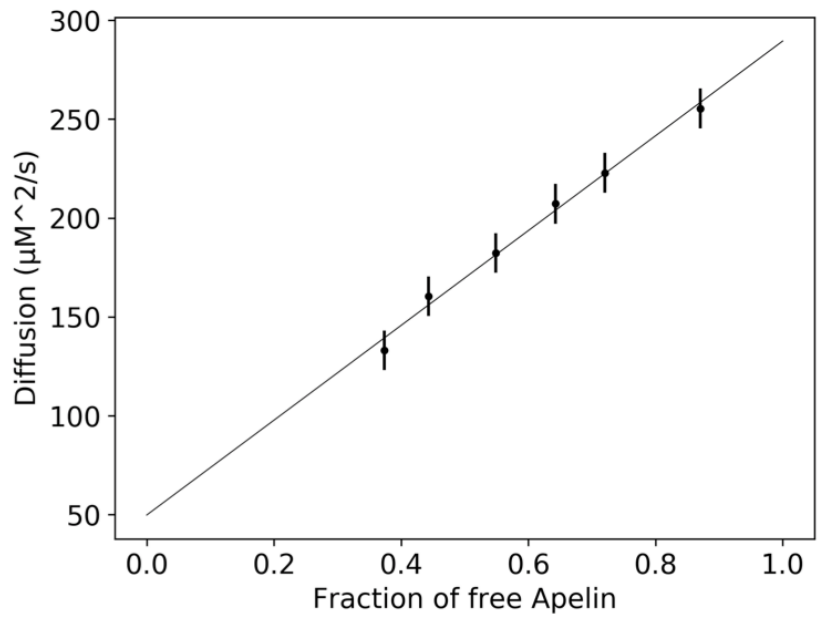
sight into the size of the F8-Ap micelles, translational diffusion coefficients were measured by ${ }^{19} \mathrm{~F}$ DOSY NMR. Values of magnetization exchange rates between the micellar and free forms of the peptide suggested a complete averaging of the measured diffusion coefficients during the $150 \mathrm{~ms}$ diffusion time of the DOSY while integration of the two trifluoromethyl resonances provided an accurate estimate of the relative populations in both monomeric and micellar states. The ${ }^{19} \mathrm{~F}$ DOSY implementing bipolar gradient pulses were measured for several concentrations of peptide ranging from 100 to $200 \mu \mathrm{M}$ where the relative fractions of micelles and free peptide undergo a large variation (Figure 4). Consistent with an exchange between two states with distinct diffusion properties, the diffusion coefficient of the free form displayed a linear dependence on the free peptide fractions, while the diffusion of added trifluoroacetic acid TFA remained constant with a value of $1230.0 \pm 96 \mathrm{\mu m}^{2} \cdot \mathrm{s}^{-1}$.

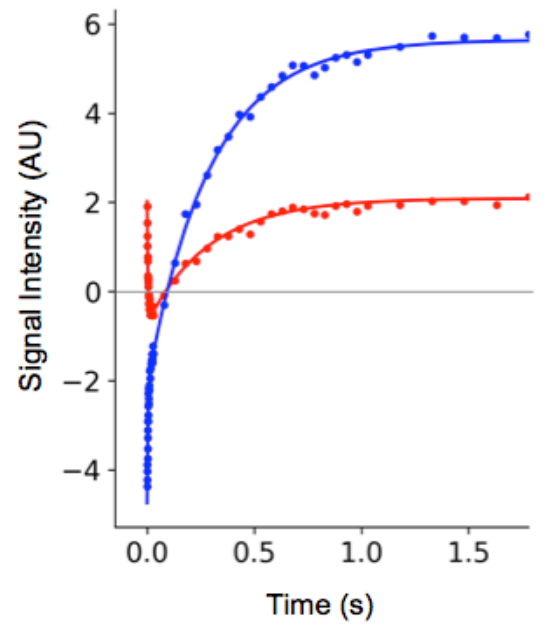

Figure 4. Diffusion coefficients of free 
fluoroapelin measured using ${ }^{19} \mathrm{~F}$ DOSY experiments for different fluoro-apelin total concentrations in PBS buffer at $298 \mathrm{~K}$.

As expected from an averaging between two states, the translational diffusion coefficient follows a linear dependence on the relative fraction of free fluoroapelin. The extrapolated values for solutions containing only monomers or micelles provided us with translational diffusion coefficients of $49.8 \pm 4$ and $289 \pm 23$ $\mathrm{um}^{2} \cdot \mathrm{s}^{-1}$. Using the Stokes-Einstein expression for the self-diffusion coefficient $D$ of a hard spherical particle of radius $\mathrm{R}_{\mathrm{h}}$ in a medium of viscosity $\eta$

$D=\frac{k_{B} T}{6 \pi \eta R_{h}}(2)$

where $\mathrm{k}_{\mathrm{B}}$ is the Boltzmann constant and $\mathrm{T}$ the temperature, a hydrodynamic radius of $49 \AA$ was obtained for the F8-Ap micelles. This value is consistent with the with Dynamic Light Scattering data measured on F8-Ap reporting a micelle's diameter of $10 \mathrm{~nm}$ (Figure S5). The ratio of diffusion coefficients obtained for the monomeric and the micellar states of F8-Ap gave a ratio of the hydrodynamic radii of $5.8 \pm 1.0$. The corresponding ratio of hydrodynamic volumes was 198, providing an estimate of the number of molecules in the micelle. It should be noted that the low peptide concentrations used in this study due to the low CMC value of F 8-Ap led to a negligible value of the volume fraction $(\Phi)$ of the micelles that usually limits the validity of equation 2 for large solute concentrations. ${ }^{[15]}$ Nevertheless, the use of the third power of the ratio of the diffusion constants is based on serious assumptions that may alter the accuracy of the aggregation factor from NMR diffusion data. First, the shape factor, assumed to be identical for the F8-Ap in the monomeric and micellar forms is probably different for a disordered peptide and an organized micelle. Second, the third power used in our calculation probably overestimates the micelle's size. Using the Flory theory for polymers, it was shown that translational diffusion coefficients measured for globular proteins scales with their size as a power of 2.56 while a power value of 1.7 was found for disordered proteins. ${ }^{[21]}$ Although slightly overestimated, the number of molecules F8-Ap is consistent with the size of the micelles. Indeed, assuming a spherical shape and a partial specific volume of $0.7 \mathrm{~cm}^{3} \cdot \mathrm{g}^{-1}$ typical of proteins led to an estimate of 172 molecules per micelle. Furthermore, such large aggregation numbers (125) were reported for single chain phospholipid Lyso PG, characterized by a low CMC (18 $\mathrm{\mu M})$ using translational diffusion measurements by NMR. ${ }^{[22]}$ For F8-Ap micelles, both translational diffusion measurements in the vicinity of CMC and size estimates from DLS suggest significantly larger aggregation numbers than those reported for the sole perfluorooctylsulfonate surfactant (36). ${ }^{[16 \mathrm{~b}]}$

In this work, we described for the first time a structural and dynamical characterization of a bioactive peptide grafted with a fluorocarbon chain at its N-terminal extremity using ${ }^{19} \mathrm{~F}$ NMR methods. On apelin-17, this addition led to self-organisational properties of the peptide into micelles of defined size with little perturbation of the peptide's structure. The self-association of F8-chain is a highly dynamic process that depends on the composition of the solution. Thereby, we suggest that fine-tuning these self-association properties based on 
${ }^{19} \mathrm{~F}$ NMR characterization could provide a novel interesting route for designing potent bioactive fluoropeptides.

Experimental Section

F8-Ap (LIT01-196) was synthetized as described in ${ }^{[12]}$. The structural characterization was performed using samples of $3 \mathrm{mg}$ and $2.4 \mathrm{mg}$ of apelin-17 and F8-Ap, respectively, dissolved either in $450 \mu \mathrm{L}$ of miliQ water and $50 \mu \mathrm{L} \mathrm{D}_{2} \mathrm{O}$ for NMR field lock. Dissolved samples were subsequently subjected to 5 minutes of vortex and 5 minutes of sonication. Resonance assignments were based on $2 \mathrm{D}$ spectra recorded at $298 \mathrm{~K}$. Through-bonds connectivities were obtained from TOCSY (80 ms mixing time) and ${ }^{1} \mathrm{H}_{-}{ }^{13} \mathrm{C}$ HSQC spectra while through-space sequential connectivities were obtained from a ROESY spectrum (300 ms mixing time) recorded on a $700 \mathrm{MHz}$ BRUKER ${ }^{\circledR}$ Avance 3 spectrometer equipped with a TCI cryogenic probe.

Self-association properties of F8-Ap were investigated by dissolving $2.6 \mathrm{mg}$ of dry peptide in $300 \mu \mathrm{L}$ of PBS buffer at $\mathrm{pH} 7.4$ to get a stock solution at 3 $\mathrm{mM}$. Cascade dilutions were performed to obtain lower concentrations, and each diluted sample was submitted to two cycles of $30 \mathrm{~s}$ sonication and $30 \mathrm{~s}$ vortex. Fluorine NMR Spectra were recorded using $3 \mathrm{~mm}$ tubes on an Avance BRUKER ${ }^{\circledR}$ spectrometer equipped with a cryogenic QCI fluorine probe, operating at 600 $\mathrm{MHz}$ proton frequency. The DOSY spectra were recorded using the bipolar-pulse pair stimulated echo pulse sequence ${ }^{[23]}$ with 24 z-gradient amplitudes up to 50 gauss. $\mathrm{cm}^{-1}$. The diffusion time and gradient pulse length were $150 \mathrm{~ms}$ and $2 \mathrm{~ms}$, respectively. The DOSY were processed using PALMA ${ }^{[24]}$ while quantifications of 1D fluorine spectra were performed using SPIKE. ${ }^{[25]}$ Modelling the fluorine signal time evolution after a selective trifluoromethyl signal inversion was performed using a non-linear least square of peak intensities using the Levenberg-Marquart algorithm implemented in the Numpy/Scipy leastsq function.

Acknowledgements

This work was supported by the Agence Nationale de la Recherche (FluoroPEP, ANR-16-CE18-0030), the French Infrastructure for Integrated Structural Biology (FRISBI, ANR-10-INSB-05-01), Instruct-ERIC, the LabEx MEDALIS (ANR-10LABX-0034), the Centre National de la Recherche Scientifique, University of Strasbourg. L.E. and S. M. R. was supported by a fellowship from the Minist'ere de l'Education Nationale and ANR.

Keywords: Fluorine NMR - GPCR ligand • Micellization • apelin-17 • peptide

[1] K. Fosgerau and T. Hoffmann, Drug Discov Today 2015, 20, 122-128. [2] H. Chen, S. Viel, F. Ziarelli and L. Peng, Chem Soc Rev 2013, 42, 7971-7982.[3] J. J. Liu, R. Horst, V. Katritch, R. C. Stevens and K. Wuthrich, Science 2012, 335, 1106-1110.[4] a) N. G. Sharaf, R. Ishima and A. M. Gronenborn, Biochemistry 2016, 55, 3864-3873; b) S. S. Stadmiller, J. S. Aguilar, C. A. Waudby and G. J. Pielak, Biophys J 2020, 118, 2537-2548.[5] G. Papeo, P. Giordano, M. G. 
Brasca, F. Buzzo, D. Caronni, F. Ciprandi, N. Mongelli, M. Veronesi, A. Vulpetti and C. Dalvit, J Am Chem Soc 2007, 129, 5665-5672.[6] A. E. Smith, L. Z. Zhou, A. H. Gorensek, M. Senske and G. J. Pielak, Proc Natl Acad Sci U S A 2016, 113, 1725-1730.[7] J. T. Gerig and R. S. McLeod, J Am Chem Soc 1973, 95, 5725-5729.[8] J. A. Hodges and R. T. Raines, J Am Chem Soc 2005, 127, 15923-15932.[9] V. Y. Torbeev and D. Hilvert, Proc Natl Acad Sci U S A 2013, 110, 20051-20056.[10] L. Malik, J. Nygaard, R. Hoiberg-Nielsen, L. Arleth, T. Hoeg-Jensen and K. J. Jensen, Langmuir 2012, 28, 593-603.[11] J. N. Francis, J. F. Thaburet, D. Bonnet, P. J. Sizer, C. B. Brown and B. Georges, Vaccine 2015, 33, 1071-1076.[12] R. Gerbier, R. Alvear-Perez, J. F. Margathe, A. Flahault, P. Couvineau, J. Gao, N. De Mota, H. Dabire, B. Li, E. Ceraudo, A. Hus-Citharel, L. Esteoulle, C. Bisoo, M. Hibert, A. Berdeaux, X. Iturrioz, D. Bonnet and C. Llorens-Cortes, FASEB J 2017, 31, 687-700.[13] K. Tatemoto, M. Hosoya, Y. Habata, R. Fujii, T. Kakegawa, M. X. Zou, Y. Kawamata, S. Fukusumi, S. Hinuma, C. Kitada, T. Kurokawa, H. Onda and M. Fujino, Biochem Biophys Res Commun 1998, 251, 471-476.[14] A. M. O'Carroll, S. J. Lolait, L. E. Harris and G. R. Pope, J Endocrinol 2013, 219, R13-35.[15] O. Södermann, P. Stilbs and W. S. Price, Concepts Magn. Reson. 2004, 23A, 121-135.[16] a) I. Huc and R. Oda, Chem. Commun. 1999, 2025-2026; b) D. Bossev, M. Matsumoto and M. Nakahara, J Phys Chem B 1999, 103, 8251-8258.[17] P. Li, J. M. Maier, E. C. Vik, C. J. Yehl, B. E. Dial, A. E. Rickher, M. D. Smith, P. J. Pellechia and K. D. Shimizu, Angew Chem Int Ed Engl 2017, 56, 7209-7212.[18] J. T. Nielsen and F. A. A. Mulder, J Biomol NMR 2018, 70, 141-165.[19] D. N. Langelaan, E. M. Bebbington, T. Reddy and J. K. Rainey, Biochemistry 2009, 48, 537-548.[20] R. J. Williams, J. N. Phillips and K. J. Mysels, Transactions of the Faraday Society 1955, 51, 728-737.[21] S. Aug'e, P.-O. Schmit, C. A. Crutchfield, M. T. Islam, D. J. Harris, E. Durand, M. Clemancey, A.-A. Quoineaud, J.-M. Lancelin, Y. Prigent, F. Taulelle and M.-A. Delsuc in NMR Measure of Translational Diffusion and Fractal Dimension. Application to Molecular Mass Measurement, Vol. 113 2009, pp. 1914-1918.[22] J. J. Chou, J. L. Baber and A. Bax, J Biomol NMR 2004, 29, 299-308.[23] D. H. Wu, A. D. Chen and C. S. Johnson, J Magn Reson 1995, 115, 260-264.[24] A. Cherni, E. Chouzenoux and M. A. Delsuc, Analyst 2017, 142, 772-779.[25] L. Chiron, M.-A. Coutouly, J.-P. Starck, C. Rolando and M.-A. Delsuc, arXiv 2016, 1608.06777.

Entry for the Table of Contents 


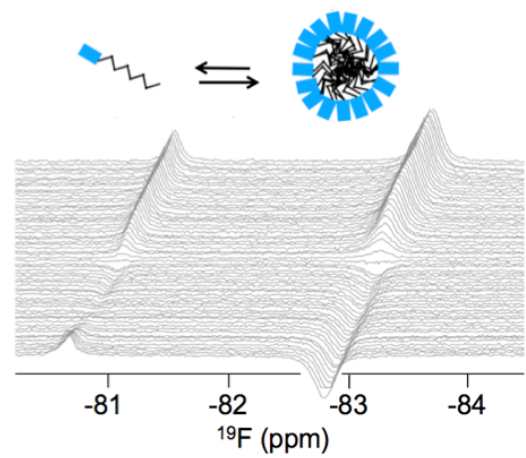

The F8-Ap, the bioactive apelin-17 peptide grafted with a fluorocarbon chain at its N-terminal extremity presents self-organisational properties. ${ }^{19} \mathrm{~F}$ NMR exchange spectroscopy and translational diffusion measurements is used to investigate this phenomenon. F8-Ap forms micelles of defined size with a fast exchange of peptides between free and micellar states. 